Reprod. Nutr. Dévelop., 1980, 20 (5 B), 1615-1619.

\title{
Données préliminaires sur la réponse des chémorécepteurs intestinaux aux acides aminés
}

par Régine JEANNINGROS, N. MEI

C. N. R. S., INP. 1, 31, chemin Joseph-Aiguier, 13274 Marseille Cedex 2.

Summary. Preliminary data on intestinal chemoreceptor responses to amino acids.

Intestinal chemoreceptor responses to amino acids in the small intestine were investigated in anaesthetized cats with a microelectrophysiological technique, i.e. vagal units in the nodose ganglion were recorded by extracellular glass microelectrodes.

About 400 neurons were tested by infusing the small intestine with a mixture of several amino acids at $38^{\circ} \mathrm{C}$. Fifty neurons, which were generally silent before the first infusion, were activated by amino acid solutions with a short latency of a few seconds. Their discharge frequency was always low (less than $10 \mathrm{~Hz}$ ). The corresponding fibres were non-medullated (conduction velocity : $0.8-1.4 \mathrm{~m} / \mathrm{s}$ ). These receptors did not respond to osmotic or mechanical stimuli. Moreover, some of them were not activated by glucose infusion and must thus be considered as specific amino acid receptors. The others which responded to both amino acids and glucose infusions were non-specific receptors. The properties of these two types of amino receptors and their roles in the nervous regulations of feeding behaviour, in particular protein satiety, are discussed.

\section{Introduction.}

Il est actuellement bien connu que l'arrivée des nutriments dans l'intestin est à l'origine de signaux qui renseignent les centres nerveux sur leur composition et leur absorption et qui participent à la régulation à court terme de la prise de nourriture (Liebling et al., 1975 ; Novin, 1976). Plusieurs résultats récents suggèrent que les propriétés rassasiantes des nutriments glucosés et protéiques reposent sur l'existence d'une chémosensibilité intestinale (Novin, Sanderson et Gonzalez, 1979 ; Vanderweele et al., 1974).

La première preuve électrophysiologique de l'existence de glucorécepteurs intestinaux vagaux a été apportée dans notre laboratoire (Mei, 1978) grâce à une technique d'enregistrement unitaire des décharges vagales provenant du tractus intestinal. En revanche, aucune donnée équivalente ne prouvait l'existence de récepteurs sensibles aux acides aminés. Or de nombreuses observations postulent l'intervention d'un mécanisme chémosensible préabsorptif (Stephens et Baldwin, 1974). De plus, le pouvoir rassasiant des acides aminés a fait l'objet de nombreuses recherches (Harper, 1976) et l'on sait actuellement que ce type de nutriments peut influencer 
le comportement alimentaire par l'intermédiaire du système nerveux extrinsèque représenté par les nerfs vagues (Vanderweele et al., 1974 ; Novin, Sanderson et Gonzalez, 1979).

Cette étude préliminaire a été réalisée en vue de répondre aux deux questions suivantes :

1. Existe-t-il au niveau de la paroi intestinale des récepteurs qui répondent à la présence des acides aminés dans la lumière intestinale?

2. Si oui, sont-ils spécifiques de ce type de nutriments ?

Pour cela, nous avons enregistré l'activité des neurones vagaux au niveau du ganglion plexiforme chez le Chat anesthésié selon la méthode mise au point au laboratoire pour l'étude systématique de la sensibilité viscérale (Mei, 1962, 1978).

\section{Matériel et méthodes.}

1. - Principe de la méthode. - L'enregistrement de l'activité unitaire des neurones vagaux a été réalisé au niveau du ganglion plexiforme droit, au moyen de micro-électrodes de verre. Le ganglion, une fois disséqué, est placé sur un support qui permet d'éviler tous les mouvements parasites (respiration, pulsations cardiaques). Le circuit d'enregistrement comprend classiquement un abaisseur d'impédance, un préamplificateur et un oscilloscope cathodique équipé d'une caméra.

Une stimulation du nerf vague est effectuée au niveau cervical afin de calculer systématiquement la vitesse de conduction des fibres sensitives. Seules les fibres amyéliniques (type $C$ ) dont la vitesse de conduction est inférieure à $2,5 \mathrm{~m} / \mathrm{s}$, sont retenues dans cette étude, car l'intestin est innervé exclusivement par cette catégorie de fibres.

2. - Animaux ef stimulations utilisés. - Les expériences ont porté sur 20 chats adultes (2 à $3 \mathrm{~kg}$ ) anesthésiés au chloralose, après une induction au Fluothane. Une portion de l'intestin d'environ $40 \mathrm{~cm}$ à partir du début du ducdénum était isolée ef perfusée en $10 \mathrm{~s}$ au moyen d'une seringue avec $30 \mathrm{ml}$ d'une des solutions suivantes: 1) mélange de 5 acides aminés : arginine, leucine, histidine, sérine et lysine à une concentration de $50 \mathrm{mM}$ chacune ou bien par un de ces acides aminés seulement $(50 \mathrm{mM}), 2)$ une solution de $\mathrm{NaCl}$ équiosmolaire, 3) une solution glucosée $1 \mathrm{M}$. Entre chaque perfusion intestinale, une vidange suivie d'un rinçage à l'eau, étaient systématiquement effectués.

Dans quelques cas, nous avons provoqué des distensions de la paroi intestinale par injection très rapide des solutions utilisées.

\section{Résultats.}

1. - Caractéristiques des récepteurs sensibles aux acides aminés. - Sur 400 neurones testés, 50 ont été activés par la perfusion intestinale du mélange d'acides aminés. En général, leur activité spontanée était nulle (fig. 1A) du moins avant la première perfusion, car après plusieurs perfusions, l'activité de base pouvait se maintenir (fig. 1B). La mise en jeu du récepteur se produit avec une latence très brève de l'ordre 
de quelques secondes, elle est reproductible et dure quelques minutes (en général de 2 à $5 \mathrm{~min}$ ) (figs. $1 \mathrm{~A}$ et $\mathrm{B}$ ).

La décharge est toujours faible (fréquence inférieure à $10 \mathrm{~Hz}$ ) et elle se poursuit après la perfusion de façon soit assez régulière, soit franchement irrégulière (bouffées de potentiels d'action). Le rinçage produit habituellement un renforcement de la décharge du récepteur de façon temporaire, suivi d'un arrêf survenant après une à deux minutes (fig. 1C).

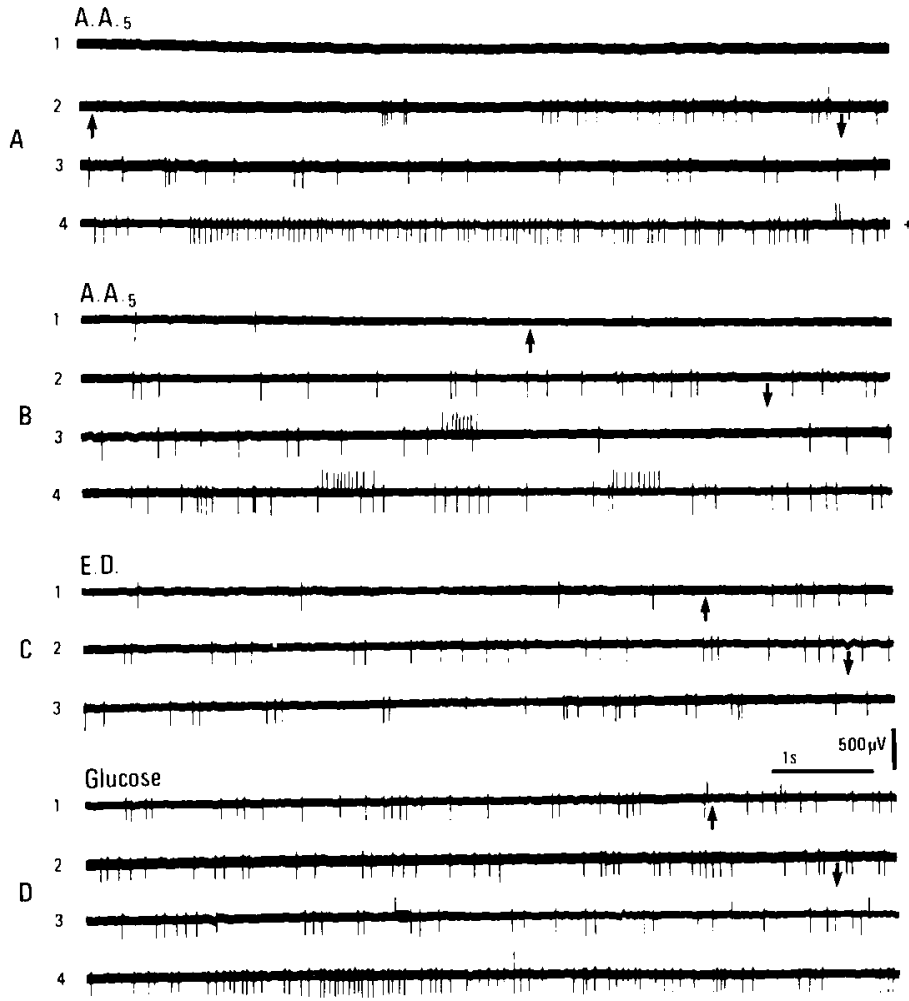

FIG. 1. - Réponse d'un chémorécepteur non spécifique à différentes perfusions intestinales.

(Les flèches indiquent le départ et la fin de la perfusion, les tracés d'une même séquence se suivent dans le temps sauf pour A4 qui a été pris 2 min après la fin de la perfusion.)

Les perfusions effectuées sont : en A et $B$ : mélange de 5 acides aminés ; en $C$ : eau ; en $D: g l u c o s e ~ 1 M$. Entre chaque test, une vidange du contenu intestinal et un rinçage d̀ l'eau étaient effectués.

Noter que : 1) La réponse du chémorécepteur (potentiels vers le bas) se produit à la fois après perfusion d'acides aminés ( $A$ et $B$ ) et après perfusion de glucose (D) ; 2) en $B$, la réponse se produit à nouveau, mais elle est moins accentuée qu'en $A$, de plus une nouvelle unité (potentiels vers le haut), qui était absente en A, apparaît ; 3) lors du rinçage (C), l'activité résiduelle est légèrement accrue.

2. - Spécificité des récepteurs. - Tous les neurones activés par les acides aminés n'étaient ni sensibles aux perfusions intestinales avec une solution de $\mathrm{NaCl}$ équiosmolaire, ni sensibles à une distension de l'intestin produite par l'injection très rapide de liquide. Ainsi, ces récepteurs ne répondent ni aux stimulus osmotiques, ni aux sti- 
mulus mécaniques. Quant à leur spécificité chimique, elle n'est pas totale pour la moitié environ des neurones testés, qui répondent également à une perfusion glucosée, comme c'est le cas dans la figure 1D. En revanche, pour l'autre moitié, on n'observait aucune réponse avec le glucose.

Par ailleurs, nous avons commencé à tester différents acides aminés individuellement ( $50 \mathrm{mM}$ chacun) dans le but de rechercher s'il existait un pouvoir discriminatif du chémorécepteur vis-à-vis de la nature de la molécule aminée. Nous n'avons pour l'instant que peu de résultats : un récepteur ayant répondu avec le mélange d'acides aminés, n'a pas été activé par l'arginine seule. Une autre unité mise en jeu par l'arginine a répondu très faiblement à l'alanine ; enfin, nous avons enregistré une unité stimulée par la leucine, mais qui restait indifférente à la lysine et à l'alanine. Des différences dans la latence d'activation étaient observées suivant l'acide aminé utilisé.

\section{Discussion.}

Ces résultats préliminaires apportent la preuve électrophysiologique de l'existence d'une chémosensibilité intestinale aux acides aminés.

1. - Caractéristiques des récepteurs aux acides aminés. - Ils sont connectés à des fibres amyéliniques vagales (type C). Leur activité spontanée, basse et même souvent nulle, ainsi que leur faible activation (fréquence de décharge inférieure à $10 \mathrm{~Hz}$ ) sont deux données communes aux glucorécepteurs intestinaux déjà connus (Mei, 1978) et semblent être une caractéristique très générale des chémorécepteurs viscéraux. De plus, cette activation précoce se produit avant que le taux sanguin des acides aminés n'augmente, ce qui exclut une localisation extra-intestinale de ces récepteurs. D'autre part, la brièveté de la latence suggère fortement une localisation de ces récepteurs proche de la lumière intestinale, probablement entre les cellules épithéliales et la voie sanguine.

2. - Spécificité des récepteurs aux acides aminés. - Il semble exister deux sortes de récepteurs :

- Un premier type qui n'est pas activé par les solutions glucosées et qui paraît, à la lumière de nos premiers résultats, être sélectif envers les différentes molécules aminées : ce sont des chémorécepteurs offrant une spécificité chimique étroite.

- Un deuxième type qui répond à deux classes de nutriments, acides aminés ef glucose. Il s'agit d'une nouvelle catégorie de récepteurs n'ayant pas encore été identifiée : les chémorécepteurs non spécifiques. Ils se distinguent des récepteurs polymodaux déjà décrits (Leek, 1972) par leur insensibilité aux stimulus mécaniques et osmotiques.

Ces nouveaux résultats viennent renforcer l'idée, encore récente, suivant laquelle la sensibilité viscérale est d'une grande complexité.

3. - Rôle supposé des récepteurs aux acides aminés. - L'existence de signaux abdominaux qui détectent les changements dans le contenu et la composition en acides aminés du bol intestinal a été souvent postulée et notamment à partir de l'éfude des mécanismes qui sous-tendent la satiété intestinale (Vanderweele et al., 1974 ; Maddison et Horrell, 1979 ; Novin et al., 1979). 
Ce travail apporte la preuve qu'il existe une chémosensibilité préabsorptive aux acides aminés intestinaux qui pouvait être responsable des signaux précédemment mentionnés. De plus, des enregistrements multiunitaires réalisés dans le VMH montrenì que l'activité de cette région est influencée par les acides aminés intestinaux (Maddison et Horrell, 1979 ; Jeanningros et Mei, résultats non publiés). D'autres travaux suggèrent l'intervention du cortex prépyriforme dans les régulations de la prise de nourriture au cours de régimes déséquilibrés en acides aminés (Leung ef Rogers, 1971). Enfin, on sait que les nerfs vagues, et en particulier le contingent amyélinique, projettent abondamment dans le VMH (Jeanningros et Mei, 1977, 1979). En fonction de ces différentes données, on peut s'attendre d'ores et déjà à ce que les récepteurs vagaux sensibles aux acides aminés soient impliqués dans les régulations nerveuses à point de départ viscéral, et en particulier dans la satiété.

Journées Ingestion-Digestion-Absorption de l'Association française de Nutrition, Paris, 15-16 novembre 1979.

\section{Références}

HARPER A. E., 1976. Protein and amino acids in the regulation of food intake, 103-113. In NOVIN D., WYRWICKA W., BRAY G., Hunger : Basic mechanisms and clinical implications, Raven Press, New York.

JEANNINGROS R., MEI N., 1977. Projections viscérales, vagales et splanchniques dans la région du noyau ventro-médian de l'hypothalamus chez le chat. C. R. Soc. Biol., 171, 831-835.

JEANNINGROS R., MEI N., 1980. Vagal and splanchnic effects at the level of the ventro-median nucleus of the hypothalamus in the cat. Brain Res., 185, 239-251.

LEEK B. F., 1972. Abdominal visceral receptors, 113-160. In NEIL E., Handbook of Sensory Physiology, vol. III/1, Enteroceptors, Springer-Verläg, Berlin, Heidelberg, New York.

LEUNG P. M.-B., ROGERS Q. R., 1971. Importance of prepyriform cortex in food intake response of rats to amino-acids. Am. J. Physiol., 221, 929-935.

LIEBLING D., EISNER J. D., GIBBS J., SMITH G. P., 1975. Intestinal satiety in rats. J. comp. physiol. Psychol., 89, 955-965.

MEI N., 1962. Enregistrement de l'activiłé unitaire des afférences vagales. Réception par microélectrodes au niveau du ganglion plexiforme. Ann. Biol. anim. Bioch. Biophys., 2, 361-364.

MEI N., 1978. Vagal glucoreceptors in the small intestine of the cat. J. Physiol., 282, 485-506.

MADDISON S., HORRELL R. I., 1979. Hypothalamic unit responses to alimentary perfusions in the anesthetized rat. Brain Res. Bull., 4, 259-266.

NOVIN D., 1976. Visceral mechanisms in the control of food intake, 357-367. In NOVIN D., WYRWICKA W., BRAY G., Hunger : Basic mechanisms and clinical implications, Raven Press, New York.

NOVIN D., SANDERSON J., GONZALEZ M., 1979. Feeding after nutrient infusions : effects of hypothalamic lesions and vagotomy. Physiol. Behav., 22, 107-113.

STEPHENS D. B., BALDWIN B. A., 1974. The lack of effect of intrajugular or intraportal injections of glucose or amino-acids on food intake in pigs. Physiol. Behav., 12, 923-929.

VANDERWEELE D. A., NOVIN D., REZEK M., SANDERSON J. D., 1974. Duodenal or hepatic portal glucose perfusion : evidence for duodenally-based satiety, Physiol. Behav., 12, 467-473. 